\title{
Effect of a perpendicular magnetic field on the shallow donor states near a semiconductor-metal interface
}

\author{
Bin Li, ${ }^{1}$ A. P. Djotyan, ${ }^{2}$ Y. L. Hao,,${ }^{1, *}$ A. A. Avetisyan, ${ }^{2}$ and F. M. Peeters ${ }^{1, \dagger}$ \\ ${ }^{1}$ Departement Fysica, Universiteit Antwerpen, Groenenborgerlaan 171, B-2020 Antwerpen, Belgium \\ ${ }^{2}$ Yerevan State University, Department of Physics, A. Manoogian 1, Yerevan 0025, Armenia \\ (Received 29 May 2012; revised manuscript received 27 November 2012; published 13 February 2013)
}

\begin{abstract}
We investigate the influence of an external perpendicular magnetic field on the lowest-energy states of an electron bound to a donor which is located near a semiconductor-metal interface. The problem is treated within the effective mass approach and the lowest-energy states are obtained through (1) the "numerically exact" finite element method, and (2) a variational approach using a trial wave function where all image charges that emerge due to the presence of the metallic gate are taken into account. The trial wave functions are constructed such that they reduce to an exponential behavior for sufficiently small magnetic fields and become Gaussian for intermediate and large magnetic fields. The average electron-donor distance can be controlled by the external magnetic field. We find that the size of the $2 p_{z}$ state depends strongly on the magnetic field when the donor is close to the interface, showing a nonmonotonic behavior, in contrast with the ground and the other excited states.
\end{abstract}

DOI: 10.1103/PhysRevB.87.075313

PACS number(s): $73.20 . \mathrm{Hb}$

\section{INTRODUCTION}

The physics of the hydrogen atom in a magnetic field attracted a lot of attention because of its considerable importance connected with the study of atoms and molecules in the intense magnetic field of neutron stars. ${ }^{1}$ The hydrogen atom in a uniform magnetic field is one of the simplest physical examples of a nonintegrable system and shows chaotic behavior. ${ }^{2}$ A large number of papers ${ }^{3-5}$ are devoted to this problem, starting from the work of Schiff and Snyder. 6

This problem became important in semiconductor physics, where due to the small effective mass and large dielectric constant, strong magnetic field effects on excitons and hydrogenlike atoms could be expected already for magnetic fields that are available in the laboratory. As is well known, Coulomb interaction changes the form of the optical absorption edge in a strong magnetic field. The peaks in the absorption occur not at the Landau energies, but at the lowest exciton levels associated with each subband. Such excitons were called diamagnetic excitons by Zakharchenya and Seysyan ${ }^{7}$ in view of their connection with diamagnetic Landau subbands. The influence of the Coulomb interaction on the nature of the absorption in a strong magnetic field was investigated in Refs. 8 and 9.

Using the method of separation of variables ${ }^{6}$ to a hydrogenlike atom in a strong magnetic field, Elliott and Loudon ${ }^{10}$ found the eigenfunctions and the eigenvalues for exciton energies in the limit of $\gamma \gg 1$ where $2 \gamma$ is the ratio of the cyclotron energy to the effective Rydberg. In Ref. 11, the problem for excited excitonic states was solved using the adiabatic approach. The theory predicts an increase of the binding energy of the discrete exciton states with increasing magnetic field. ${ }^{12}$ Due to the similarity between hydrogenlike and excitonic states, an enhancement of the binding energy takes place also for impurity states.

A doping with impurities in concentrations close to the critical value "suppresses" the Coulomb interaction and reduces the excitonic absorption. The quenching of the exciton absorption in germanium due to the screening of the Coulomb interaction by free carriers was observed in Ref. 13.
Reappearance of the excitonic absorption takes place in a strong magnetic field.

This problem was theoretically investigated in Ref. 14, where it was found that the extension of the exciton ground state along the magnetic field decreases with increasing magnetic field. Due to this, the destroyed excitonic states reconstruct in a magnetic field.

Simple approximations suffice to obtain analytical results for exciton and hydrogenlike atom binding energy in weak and strong magnetic fields. For intermediate magnetic field, no analytic exact results are available and one has to rely on approximations or pure numerical approaches. In strong magnetic fields, accurate analytical results could be obtained by using the adiabatic approach. ${ }^{10,12}$

Renewed interest in the energetics of hydrogenlike atoms in a magnetic field appeared with the investigation of the energy spectrum of shallow donors in confined systems, e.g., a quasi-two-dimensional (Q2D) system in a magnetic field ${ }^{15}$ as well as with the study of dopant atoms in semiconductor devices near different interfaces in the presence of external fields. ${ }^{16}$ In Ref. 17, a theoretical study of the electronic and magnetic properties of the Mn-induced acceptor level obtained by substituting a single $\mathrm{Ga}$ atom in the surface layer of GaAs was presented. It was shown that the proximity of the $\mathrm{Mn}$ atoms to the surface must have a significant impact on the magnetic anisotropy energy of the system which was previously theoretically investigated in Ref. 18 for bulk GaAs.

Recently, the problem of a gated donor has attracted a lot of attention due to the perspective of atomic-scale electronics. A donor located close to the oxide-silicon interface has been proposed as a possible realization of the Kane qubit architecture. ${ }^{19}$ In contrast to bulk donors, it is possible to adiabatically pull the donor electron to the interface, and to exert precise quantum control. Aside from applications in quantum computing, discrete dopants become very important for diverse applications in nanoscale electronics. In Ref. 20, the possibility of the creation of a novel device based on single-dopant functionality was studied, and single-electron 
pumping via a number of individual tunable donors located between a set of gates in a nanowire was reported.

The charging energies of $D^{0}$ and $D^{-}$in silicon as a function of applied electric field and donor depth was calculated in Ref. 21 using the self-consistent field tight-binding method, and it was shown that the results were in good agreement with experimental measurements. Results based on the effective mass approach can be found in Ref. 22.

For a single donor $P$ in $\mathrm{Si}$, close to a $\mathrm{SiO}_{2}$ interface, the possibility of donor-bound electron manipulation, i.e., onequbit operation between the donor and the interface by electric and magnetic fields, was investigated in Ref. 23. One of the problems for quantum computation in doped Si emerges from the incomplete control of the exact position of the donors and therefore from the indetermination of the value of the exchange energy between two neighboring donor electrons. Recently, full spatial control of the incorporation of single $P$ dopant atoms in Si was realized in Ref. 24, which opens a new possibility for the creation of atomic-scale electronic devices. In a more recent study (Ref. 25), individual $\mathrm{Mn}$ atoms were carefully positioned on the GaAs surface and on the first few layers below.

On the other hand, exchange control can also be realized by applying a magnetic field perpendicular to the interface, which squeezes the electron wave function and, as a result, controls the overlap of the electron wave function of neighboring dopant atoms. So, the problem of the energetics of a single dopant near a semiconductor-metal interface in a magnetic field becomes a problem of great interest both from a theoretical as well as from an experimental point of view.

In the absence of a magnetic field, the energy spectrum of a shallow donor located near a semiconductor-metal interface was investigated in Ref. 26 and in a gated semiconductor nanowire ${ }^{27}$ by using the finite element method (FEM). It was found that the dielectric mismatch effect has a very large effect when the donor approaches the interface. In Ref. 28, we developed a variational approach for shallow donor states localized in a semiconductor near a semiconductor-insulatormetal interface. We introduced a term in the variational wave function which describes the interaction of the electron with the images in the insulator and/or in the metallic gate which leads to a considerable lowering of the energy, especially when the impurity is located very near the interface. Our variational results for the ground- and the first-excited-state energies of the impurity electron were found to be in good agreement with the energies found using FEM.

It is well known that a strong magnetic field affects the energy spectrum of impurity states. In this paper, we extend our previous work and investigate the energy spectrum of a shallow donor near a semiconductor-metal interface in the presence of a magnetic field perpendicular to the interface. Since the Schrödinger equation for a hydrogenlike system in a magnetic field can not be solved analytically, we resort to a variational approach. Our variational calculation is an extension of the variational approach presented in Ref. 28 where we include now a factor which is responsible for magnetic field effects. Similar as in Refs. 15 and 29, the wave function exhibits an exponential behavior for small magnetic fields but becomes Gaussian for large magnetic fields. To get a feeling of the accuracy of our variational results, we also present "exact" numerical calculations based on FEM.

This paper is organized as follows. In Sec. II, we propose trial wave functions for the ground as well as for excited states for an impurity electron near a semiconductor-metal interface in the presence of a perpendicular magnetic field. The discussion of the results obtained on the basis of our variational approach together with the "exact" FEM results are given in Sec. III.

\section{TRIAL WAVE FUNCTIONS}

In this section, we consider a donor at a semiconductorinsulator-metal interface in the presence of an external magnetic field. When a constant magnetic field $\mathbf{B}=(0,0, B)$ with $\mathbf{A}=\frac{1}{2}[\mathbf{B} \times \mathbf{r}]\left(A_{\varphi}=H \rho / 2, A_{\rho}=A_{z}=0\right)$ is applied along the $z$ axis, the Hamiltonian for the impurity electron in cylindrical coordinates becomes

$$
\begin{aligned}
H= & -\frac{\hbar^{2}}{2 m_{\perp}}\left[\frac{\partial^{2}}{\partial \rho^{2}}+\frac{1}{\rho} \frac{\partial}{\partial \rho}+\frac{1}{\rho^{2}} \frac{\partial}{\partial \varphi^{2}}+\sigma \frac{\partial^{2}}{\partial z^{2}}\right] \\
& -\frac{i \hbar \omega_{c}}{2} \frac{\partial}{\partial \varphi}+\frac{m_{\perp} \omega_{c}^{2} \rho^{2}}{8}+U_{c}(\vec{r}),
\end{aligned}
$$

where $\omega_{c}=e B / m_{\perp} c$ is the cyclotron frequency, $m_{\perp}$ is the transverse effective mass, and $\sigma$ is the ratio between the transverse and longitudinal $m_{\|}$effective masses. As in Ref. 26, we assume that the oxide layer is very thin and its only effect is to provide a very high potential barrier for the electron, i.e., to prevent the electron from penetrating into the metal. The thin oxide layer does not contribute to any dielectric mismatch, and only the metallic gate screens the Coulomb potential. The potential energy $U_{c}(\vec{r})$ between the electron and the impurity near the semiconductor-metal interface, at a position $\vec{r}_{d}=(0, d)$, is given by ${ }^{26}$

$$
U_{c}(\vec{r})=\frac{e^{2}}{\varepsilon_{s}}\left[-\frac{1}{4 z}+\frac{1}{\sqrt{\rho^{2}+(z+d)^{2}}}-\frac{1}{\sqrt{\rho^{2}+(z-d)^{2}}}\right],
$$

with $\rho=\sqrt{x^{2}+y^{2}}$, and $\varepsilon_{s}$ is the dielectric constant at the semiconductor side of the interface. The dielectric constant for typical semiconductors of interest is about 10, while it is infinite in the metal. The dielectric mismatch effects at the interface give rise to image charges that screen the potential between the electron and the impurity. In Eq. (2), the first term describes the attractive interaction between the electron and its image, the second term is due to the repulsive interaction between the electron and the donor image (as well as between the donor and the electron image), and the last term represents the normal impurity-electron interaction. In dimensionless units expressed in terms of the Bohr radius $a_{B}=$ $\hbar^{2} \varepsilon_{s} / m_{\perp} e^{2}$ and twice the Rydberg energy $2 R^{*}=\hbar^{2} / m_{\perp} a_{B}^{2}$, the Hamiltonian of the system transforms to

$$
\begin{aligned}
H=- & \frac{1}{2}\left[\frac{\partial^{2}}{\partial \rho^{2}}+\frac{1}{\rho} \frac{\partial}{\partial \rho}+\frac{1}{\rho^{2}} \frac{\partial}{\partial \varphi^{2}}+\sigma \frac{\partial^{2}}{\partial z^{2}}\right] \\
& +\frac{\gamma L_{z}}{2}+\frac{\gamma^{2} \rho^{2}}{8}+U_{c}(\vec{r}),
\end{aligned}
$$


where $L_{z}=-i \partial / \partial \varphi$ is the $z$ component of the angular momentum operator, and the dimensionless parameter of magnetic field $\gamma=a_{B}^{2} / a_{H}^{2}$ with magnetic length $a_{H}=(\hbar c / B e)^{1 / 2}$. We use $\sigma=1$ in this paper. In GaAs, we have $a_{B}=$ $9.89 \mathrm{~nm}, 2 R^{*}=11.6 \mathrm{meV}$, and $\varepsilon_{s}=12.9$, while for $\mathrm{Si}$ with $m_{\perp}=0.19 m_{0}$ we have $a_{B}=3.31 \mathrm{~nm}, 2 R^{*}=36.5 \mathrm{meV}$, and $\varepsilon_{s}=11.9$.

In the presence of a magnetic field, we choose the variational wave function for the ground $1 s$ as well as for $2 p_{-}$and $2 p_{+}$states of the system as

$$
\psi=N z g[\rho,(z-d)] f(z) \exp \left(-\delta \rho^{2} / 4\right) \rho^{|m|} \exp (\operatorname{im} \varphi),
$$

where the factor $z$ guarantees that the wave function satisfies the boundary condition $\psi(z=0)=0 ; m=0$ corresponds to the ground state and $m=-1(+1)$ corresponds to the $2 p_{-}\left(2 p_{+}\right)$state. The exponential factor $f(z)=\exp (-\beta z)$ describes the effect of image charges, ${ }^{28}$ and $g[\rho,(z-d)]=$ $\exp \left[-\lambda \sqrt{\rho^{2}+(z-d)^{2}}\right]$ is responsible for the electron-donor interaction; $\lambda, \beta$ are variational parameters. In the trial function (4), instead of the magnetic factor $\exp \left(-\gamma \rho^{2} / 4\right)$ (which corresponds to the ground-state function in the absence of the impurity and interface), we introduced a third variational parameter $\delta$ through the factor $\exp \left(-\delta \rho^{2} / 4\right)$ which is crucial to describe the large magnetic field behavior. For weak magnetic fields, better results are obtained using a trial function with an exponential behavior which takes into account the magnetic field induced asymmetry, namely, when two additional variational parameters $\lambda_{1}$ and $\lambda_{2}$ are included, and the value of $\gamma$ is fixed:

$$
\begin{aligned}
\psi= & N z \exp \left[-\sqrt{\lambda_{1}^{2} \rho^{2}+\lambda_{2}^{2}(z-d)^{2}}\right] \exp (-\beta z) \\
& \times \exp \left(-\gamma \rho^{2} / 4\right) \rho^{|m|} \exp (\operatorname{im} \varphi) .
\end{aligned}
$$

The normalization constant is $N=\sqrt{1 / 2 \pi A}$ with $A=$ $\int_{0}^{\infty} \rho d \rho \int_{0}^{\infty} d z|\psi|^{2}$.

Using the trial function given by Eq. (4) with $m=0$, we obtain the following expression for the ground-state energy:

$$
E_{1 s}=\gamma / 2+\left(C+T_{1}+T_{2}+T_{3}\right) / A,
$$

where

$$
\begin{aligned}
C= & \int_{0}^{\infty} \rho d \rho \int_{0}^{\infty} d z U_{c}(\rho, z) f_{1}^{2}(z) \zeta(\rho, z) \\
T_{1}= & \int_{0}^{\infty} \rho d \rho \int_{0}^{\infty} d z\left(\frac{\lambda}{\mu}-\frac{\lambda^{2}}{2}\right) f_{1}^{2}(z) \zeta(\rho, z), \\
T_{2}= & \int_{0}^{\infty} \rho d \rho \int_{0}^{\infty} d z\left(\frac{\lambda \delta}{2 \mu}-\frac{\gamma^{2}}{8}+\frac{\delta^{2}}{8}\right) \rho^{2} f_{1}^{2}(z) \zeta(\rho, z), \\
T_{3}= & \int_{0}^{\infty} \rho d \rho \int_{0}^{\infty} d z \\
& \times\left(\frac{\lambda^{2}(z-d)}{\mu} \frac{\partial f_{1}(z)}{\partial z}-\frac{1}{2} \frac{\partial^{2} f_{1}(z)}{\partial z^{2}}\right) f_{1}(z) \zeta(\rho, z),
\end{aligned}
$$

with $\quad \mu=\sqrt{\rho^{2}+(z-d)^{2}}, \quad \zeta(\rho, z)=g[\rho,(z-d)]^{2}$ $\exp \left(-\delta \rho^{2} / 2\right)$, and $f_{1}(z)=z \exp (-\beta z)$.
Now we consider the $2 p_{z}$ and $2 s$ exited states. In the presence of a weak magnetic field, the trial function for the $2 p_{z}$ state is chosen as a product of the $1 s$ wave function [Eq. (4) with $m=0]$ and adding the factor $(z-d),{ }^{30}$ i.e., as

$$
\psi=N z(z-d) g[\rho,(z-d)] \exp \left(-\delta \rho^{2} / 4\right) f(z) .
$$

The $2 s$ state wave function, in the region of weak magnetic fields, is chosen as a product of the $1 s$ wave function and adding the factor $(1-\alpha r)=\left[1-\alpha \sqrt{\rho^{2}+(z-d)^{2}}\right]$,

$$
\psi=N z(1-\alpha r) g[\rho,(z-d)] \exp \left(-\delta \rho^{2} / 4\right) f(z),
$$

where the value of $\alpha$ is defined from the orthogonalization condition of the $1 s$ and $2 s$ wave functions. ${ }^{28,30}$

For intermediate and strong magnetic fields with $\gamma \geqslant 1$, the Coulomb interaction of the electron with the donor is expected to be almost one dimensional, we use the adiabatic approach ${ }^{10}$ and choose the trial function for the $2 p_{z}$ state as

$$
\psi=N z(z-d) f(z) \exp \left(-\delta \rho^{2} / 4\right),
$$

where the interaction of the electron with the donor as well as with the images is described now only by one factor $f(z)=$ $\exp (-\beta z) ; \beta$ and $\delta$ are variational parameters.

Similarly, for the $2 s$ state $^{28}$ in the presence of a strong magnetic field, we chose the trial function

$$
\psi=N z(1-\alpha z) f(z) \exp \left(-\delta \rho^{2} / 4\right) .
$$

The exponential factor $f(z)=\exp (-\beta z)$ describes the effect of the image charges ${ }^{28}$ and is responsible here also for the electron-donor interaction which for a strong magnetic field is assumed one dimensional (the validity of this assumption will be justified further through a comparison with the FEM results for the probability density).

It is well known that the adiabatic approach for the ground-state energy is valid for large magnetic fields with $\gamma>1$, where the magnetic length becomes smaller than the effective Bohr radius of the impurity electron. Notice that for excited states with larger extent of the wave function, the adiabatic approach becomes accurate already in relatively weak magnetic fields. ${ }^{29}$

The binding energy is defined as the difference between the energy of the system in the absence of the Coulomb interaction and the total energy. For the $1 s, 2 p_{-}, 2 s$, and $2 p_{z}$ states, the binding energy is

$$
E_{b, i}=\gamma / 2-E_{i},
$$

where $\gamma / 2$ is the energy of the first Landau level and $E_{i}$ is the total energy of the corresponding state. For the $2 p_{+}$state, we have $^{29}$

$$
E_{b, 2 p_{+}}=3 \gamma / 2-E_{2 p_{+}} .
$$

\section{NUMERICAL RESULTS}

The numerical results obtained for the ground-state energy using the wave function given by Eq. (4) with three variational parameters $(\lambda, \beta, \delta)$ as well as the results with the same function but with $\delta$ fixed to the value of $\gamma$ (i.e., using two variational parameters $\lambda, \beta)$ in the case of equal transverse and longitudinal effective masses $(\sigma=1)$ are shown in Fig. 1 by symbols for distance $d=2 a_{B}$ between the donor and the 


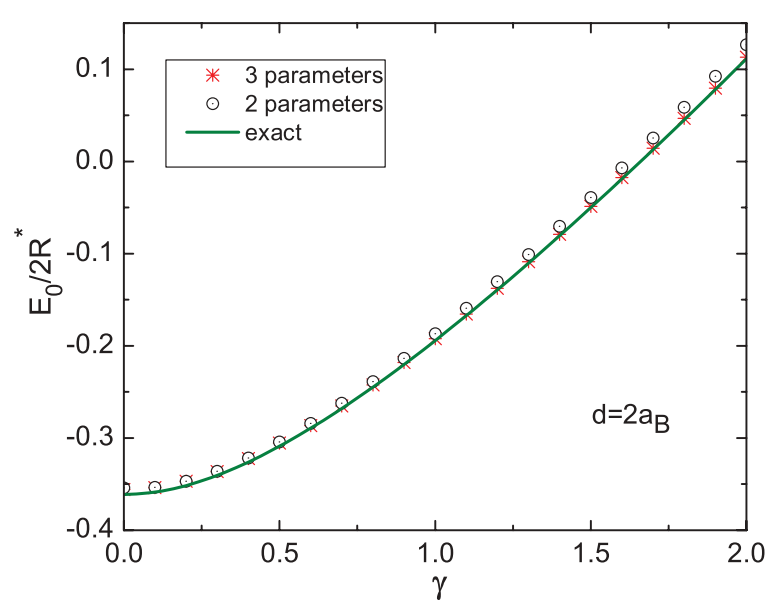

FIG. 1. (Color online) The dependence of the ground-state energy (in units of $2 R^{*}$ ) as a function of the magnetic field parameter $\gamma=$ $a_{B}^{2} / a_{H}^{2}$ for the donor position $d / a_{B}=2$ with respect to the metal interface. We compare the results obtained using the three-variationalparameter trial function (4) (stars) with the results when only two variational parameters are included (circles) and with the "exact" FEM result (solid line).

interface. In the same figure, we also show the "numerical exact" FEM results by solid curve.

For weak magnetic fields (i.e., small $\gamma$ ) and for $d=2 a_{B}$, the maximum relative error between the exact and variational energies is about $1.5 \%$ (for $\gamma=0.2$ ). The variational ground-state energy for $\gamma=0.2$ is $E_{0} / 2 R^{*}=-0.3469$ using the wave function (4) where we fixed $\delta=\gamma=\hbar \omega_{c} / 2 R^{*}$, while the exact result is $E_{0} / 2 R^{*}=-0.3515$. The variational function (5) gives lower energy $E_{0} / 2 R^{*}=-0.3481$ and the relative difference with FEM is less than $1 \%$.

For strong magnetic fields, e.g., for $\gamma=2\left(d=2 a_{B}\right)$, we found $E / 2 R^{*}=0.1114,0.1129,0.1267$ for the exact result, three-parameter Eq. (4) trial function (i.e., with $\lambda, \beta, \delta$ ), and when using two parameters $\lambda, \beta$ in Eq. (4), respectively. Notice that now it is important to consider $\delta$ as a variational parameter which reduces the relative error from $10 \%$ (when $\delta=\gamma$ ) to $1.5 \%$.

Next we consider the excited states. Figures 2(a) and 2(b) show the energy of the few lowest states, namely, $2 p_{-}, 2 p_{+}$, $2 s$, and $2 p_{z}$ for $d=2 a_{B}$ and $10 a_{B}$, respectively, which can be labeled by the quantum numbers $(n, l, m)$ (in terms of hydrogeniclike atoms, which is valid only in weak magnetic fields). As a result of the cylindrical symmetry, only the azimuthal quantum number $m$, i.e., the quantum number of the angular momentum operator $L_{z}$, is well defined. The states $(1,0,0),(2,0,0),(2,1,0),(2,1,-1)$, and $(2,1,1)$ correspond to the $1 s, 2 s, 2 p_{z}, 2 p_{-}$, and $2 p_{+}$hydrogenlike states, respectively.

Figure 2(a) shows the dependence of the excited-state energies (in units of $2 R^{*}$ ) as a function of the magnetic field parameter $\gamma=a_{B}^{2} / a_{H}^{2}$ for the donor distance $d=2 a_{B}$ from the metallic gate for $2 p_{+}$state (dotted-dotted-dashed curve), $2 p_{-}$state (dotted-dashed curve) calculated on the basis of FEM as well as variationally using the three-parameter wave function [Eq. (4)] (variational results are indicated by stars). The FEM results obtained for the $2 s$ state are shown by the dashed line, and the results obtained variationally using the
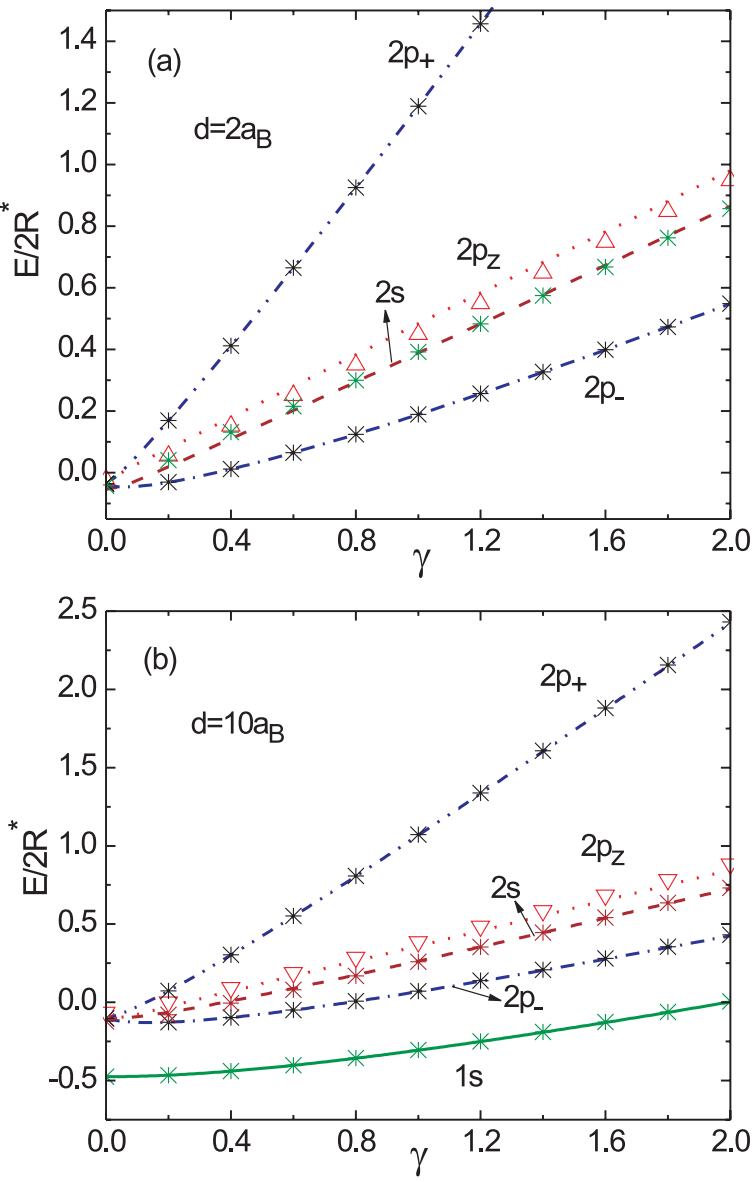

FIG. 2. (Color online) The dependence of the few lowest state energies (in units of $2 R^{*}$ ) on the magnetic field parameter $\gamma=$ $a_{B}^{2} / a_{H}^{2}$ for the $2 p_{+}$state (dotted-dotted-dashed curve), $2 p_{-}$state (dotted-dashed curve), $2 s$ (dashed curve), and $2 p_{z}$ (dotted curve) states calculated by FEM and variationally (symbols). Here, (a) is for the case $d=2 a_{B}$ and (b) is for $d=10 a_{B}$.

trial function (10) are presented by stars; the "exact" energy of the $2 p_{z}$ state is shown as a dotted line, and the variational results with Eq. (9) are indicated by triangles.

For weak magnetic field with $\gamma=0.2$ and for a donor located at $d=2 a_{B}$ using the three- parameter $(\lambda, \beta, \delta)$ variational function given by Eq. (4), we obtain energies (in units $2 R^{*}$ ) $E_{2 p_{-}}=-0.0302$ and $E_{2 p_{+}}=0.1697$, while the exact results for these states are $E_{2 p_{-}}=-0.031, E_{2 p_{+}}=0.169$ (relative error is about $2.5 \%$ for the $2 p_{-}$state and less than $1 \%$ for $2 p_{+}$). The trial function (4) leads to better results in strong magnetic fields, e.g., the relative error is about $0.2 \%$ for $\gamma=2$; we obtain variationally $E_{2 p_{-}}=0.5491$ and $E_{2 p_{+}}=2.5491$, while the exact results are $E_{2 p_{-}}=0.5485, E_{2 p_{+}}=2.5484$.

In intermediate and strong magnetic fields, the results obtained for the $2 s$ state (see Fig. 2) using the trial function (10) with the one-dimensional factor $f(z)$ (that is responsible also for the electron-donor interaction) are close to the FEM results (dashed line) starting from $\gamma=0.6$ with relative error of about $1 \%$. We found that for the excited states, the adiabatic approximation, as is noticed in Ref. 29, is accurate for intermediate and large values of $\gamma$. In weak magnetic fields, a better agreement of the variational results with the FEM ones 


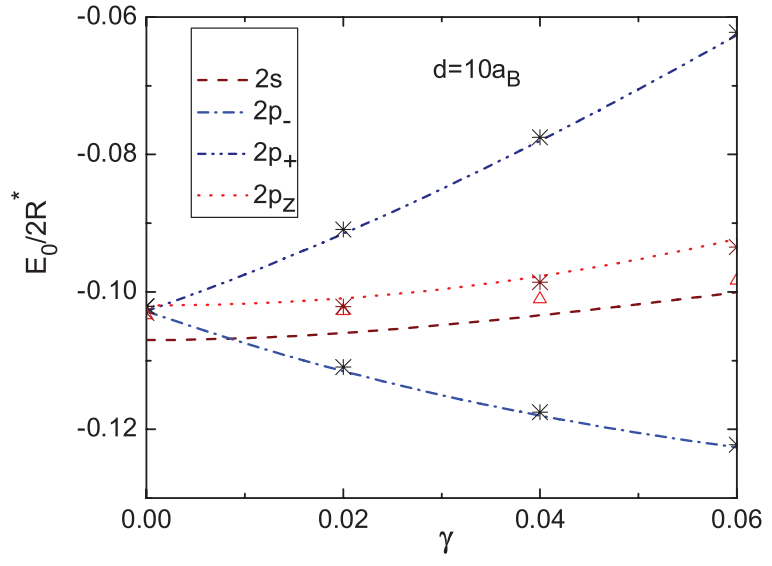

FIG. 3. (Color online) The dependence of the excited-state energies (in units of $2 R^{*}$ ) for small values of the magnetic field parameter $\gamma$; FEM results: dotted-dashed line for the $2 p_{-}$and dotted-dotted-dashed curve for the $2 p_{+}$; the $2 s$ state is presented by dashed line and the $2 p_{z}$ by dotted line. The variational results are presented by symbols (triangles are for the $2 p_{z}$ state and stars for the other states). The distance of the donor from the metal-semiconductor interface is $d=10 a_{B}$.

for the $2 s$ state is obtained using the trial function (8) with the three-dimensional hydrogenlike factor $g[\rho,(z-d)]$ as shown in Fig. 3.

When a magnetic field with $\gamma>0.8$ is applied, we find that the difference between the variational results [on the basis of the trial function (9)] and the FEM results for the $2 p_{z}$ state energy is about $5 \%$ (and much larger for the binding energy). For weak magnetic fields and for large values of $d$, good results for the $2 p_{z}$ state are found with the trial function (7) with an error less than $8 \%$ (Fig. 3).

Figure 2(b) shows that for the case of $d=10 a_{B}$, the lowest four excited states in the absence of magnetic field are almost degenerate since the influence of the interface and the metallic gate is weak for large values of $d$. The magnetic field lifts the degeneracy between these four states.

The situation in the case of $d=2 a_{B}$ is quite different due to the more pronounced screening effect of the metallic gate and the repulsion of the interface due to the requirement of $\psi(z=$ $0, \rho)=0$. One can see in Fig. 2(a) that the $2 s$ and $2 p_{z}$ states have noticeably different energies as compared with the $2 p_{ \pm}$ states even for zero magnetic field, where the latter two states are still degenerate. The $2 p_{+}$and $2 p_{-}$states start to split from each other when a magnetic field is applied. When we further increase the magnetic field, due to the Zeeman energy the $2 p_{-}$state has a smaller energy than $2 s$. As expected, for large values of $d$ the ground state and the four lowest-energy states tend to the hydrogenic energy levels $E_{n}=\hbar^{2} /\left(2 m_{e} a_{B}^{2} n^{2}\right)$ with decreasing magnetic field.

In order to study the magnetic field dependence of the electron binding energy including the influence of the metallic interface, we present in Figs. 4(a) and 4(b) the "exact" FEM numerical results together with the variational ones obtained using Eqs. (11) and (12), correspondingly, for $d=2 a_{B}$ and $10 a_{B}$. We found that for both cases, the binding energy increases with increase of magnetic field and a stronger dependence is observed for the states with lower energy.
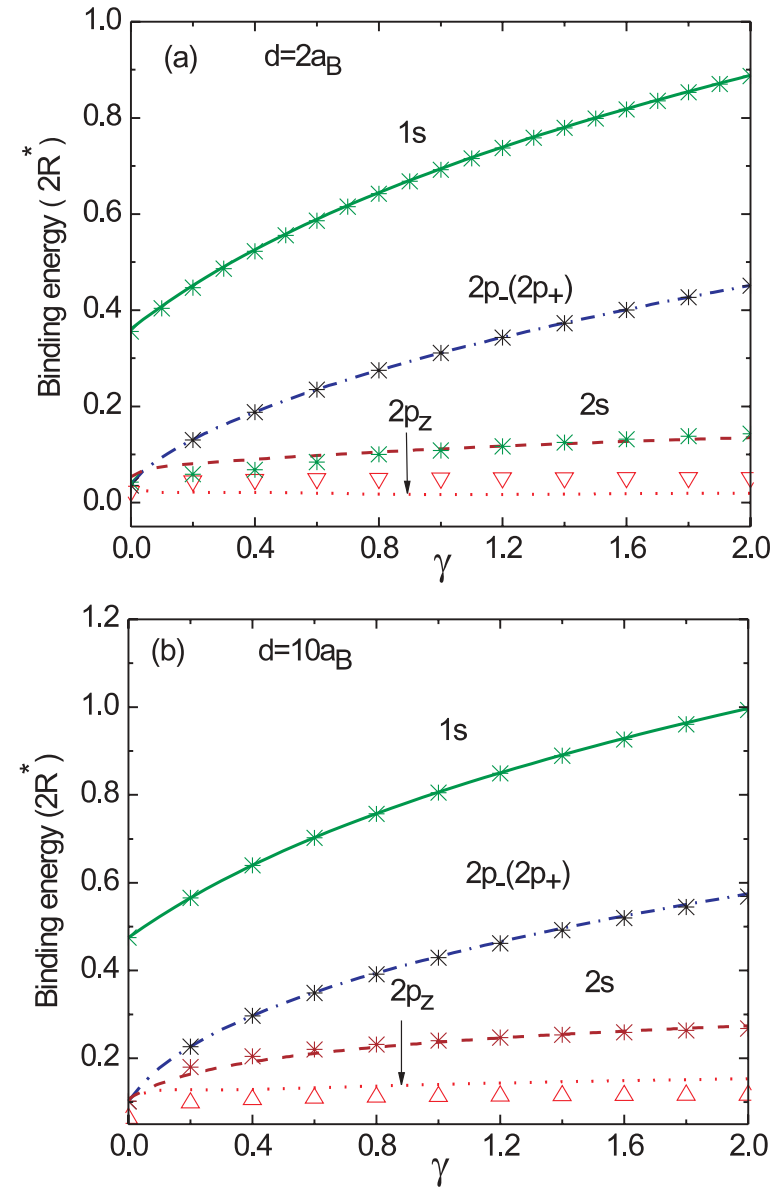

FIG. 4. (Color online) The dependence of the binding energy (in units of $2 R^{*}$ ) for the ground $1 s$ (solid line) and excited $2 p_{-}\left(2 p_{+}\right)$ (dotted-dashed curve), $2 s$ (dashed curve), and $2 p_{z}$ (dotted curve) states as a function of the magnetic field parameter $\gamma$ calculated by FEM, and corresponding variational results are indicated by stars for $1 s, 2 s, 2 p_{-}\left(2 p_{+}\right)$states and by triangles for the $2 p_{z}$ state when (a) $d=2 a_{B}$ and (b) $d=10 a_{B}$.

Figure 4(a) shows that the binding energy of the ground state calculated variationally and by FEM are very close to each other. The relative error is even less than the corresponding error calculated for the total energy; for $\gamma=0.2, d=2 a_{B}$ the error is now $1 \%$, and for strong fields $(\gamma=2)$ it is much less than $1 \%$. The binding energy for the $2 p_{-}$and $2 p_{+}$states are equal to each other (the $2 p_{-}$state is under the first Landau level, while the $2 p_{+}$state energy tends to the $3 \gamma / 2$ in strong magnetic fields with $\gamma \gg 1$ ). Similarly as for the $1 s$ state, the relative error for the binding energies of the $2 p_{-}\left(2 p_{+}\right)$states is less than $1 \%$. Good agreement between variational and FEM results is also obtained for the $2 s$ state binding energy. In contrast, for the $2 p_{z}$ state, the relative error is much larger than the corresponding error obtained for the total energy. The fact that the result of the variational method for $2 p_{z}$ is rather different from that of the FEM can be explained by the shallowness of this state, and the variational method gives less accurate results for the binding energy.

The probability densities of the $2 s$ (bottom four figures) and $2 p_{z}$ (top four figures) levels in the absence of magnetic field $(\gamma=0)$, in weak $(\gamma=0.1$ and 0.2$)$ and in large 


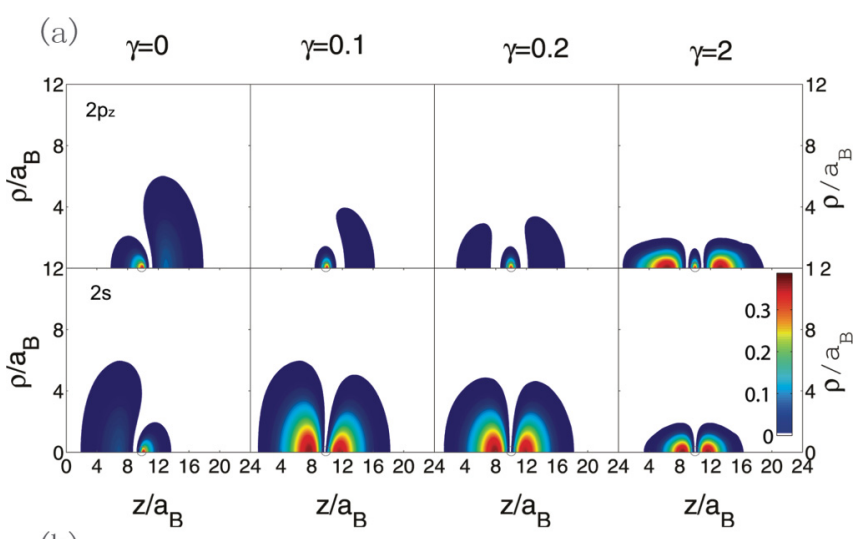

(b)

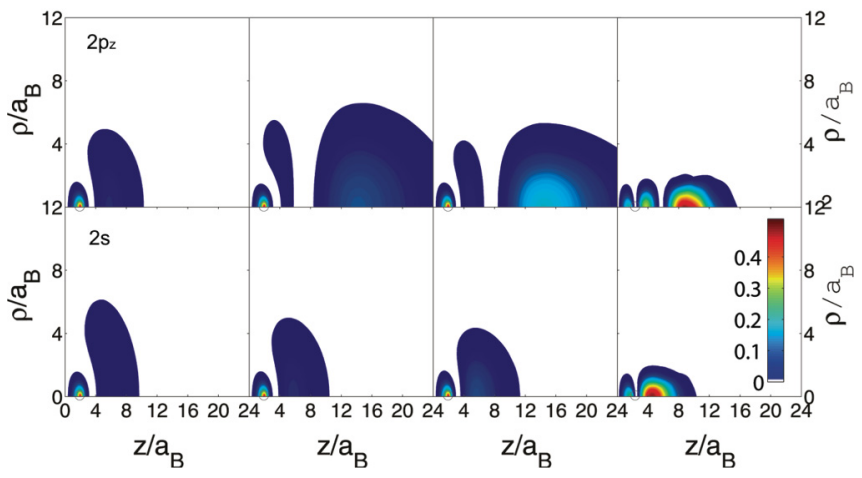

FIG. 5. (Color online) Probability density of the electron for four different values of $\gamma=0,0.1,0.2,2$ in the $\rho-z$ plane, where the top (bottom) four figures correspond to the densities of the $2 p_{z}(2 s)$ energy level for (a) $d=10 a_{B}$ and (b) $d=2 a_{B}$. The donor positions are indicated by the white circle. Blue (red) areas represent low (high) probabilities.

$(\gamma=2)$ magnetic field for donor distance $d=10 a_{B}$ and $2 a_{B}$, are shown in Figs. 5(a) and 5(b), respectively. We did not plot the probability densities of the $2 p_{-}\left(2 p_{+}\right)$and $1 s$ levels since the shapes of the wave functions do not differ significantly. For a better understanding of the influence of the magnetic field on the different energy levels, we calculated the effective radius $\langle\rho\rangle$ of the electron and the average distance from the donor $\langle z-d\rangle$. The results for the case of $d=10 a_{B}\left(d=2 a_{B}\right)$ are shown in Fig. 6 (Fig. 7). The change of the shape of the density probability in Fig. 5 can be explained in the following manner. In the absence of the interface, the electron probability density for the $2 p_{z}$ state is prolonged in the $z$ direction, while the $2 s$ state is spherical. Due to the shape of the $2 p_{z}$ wave function, the interface and the metallic gate tend to repel the electron stronger in the $2 p_{z}$ state than in the $2 s$ state. In the absence of magnetic field, in Fig. 5(a) we see that in the $2 p_{z}$ state for $d=10 a_{B}$ the left lobe is pushed towards the donor, while the right one has a much larger extend in the $\rho$ direction. Correspondingly, in Fig. 6 we have positive values for average distance $\langle z-d\rangle$. Such configuration of the second lobe occurs due to the fact that the electron prefers to be far away from the donor image and interact stronger with its image.

For the $2 s$ state (for $\gamma=0$ ), the maximum of the electron density is found at the location of the donor, and the outer shell of the electronic density is shifted to the interface, leading to a negative value for the average $\langle z-d\rangle$ as shown in Fig. 6 .

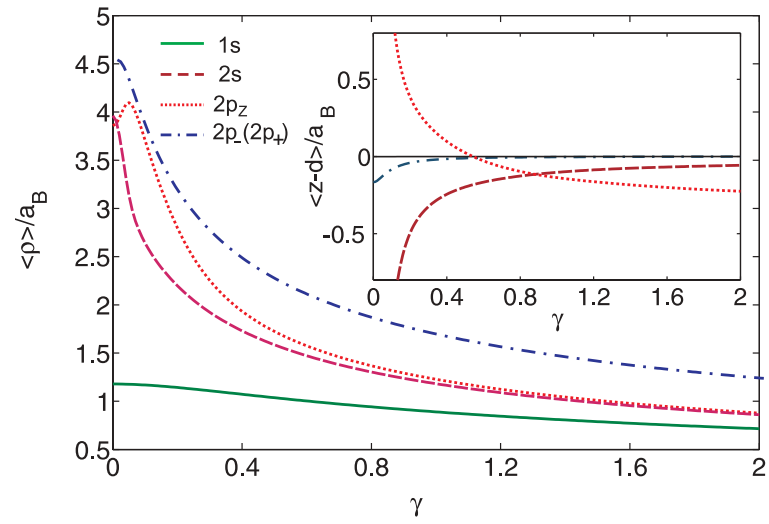

FIG. 6. (Color online) Effective radius $\langle\rho\rangle$ of the electron, in the case of large donor distance $d=10 a_{B}$, as a function of magnetic field $\gamma$, for five different energy levels: $1 s$ (solid curve), $2 s$ (dashed curve), $2 p_{z}$ (dotted curve), and $2 p_{-}\left(2 p_{+}\right)$(dotted-dashed curve). Inset: average distance of the electron from the donor $\langle z-d\rangle$ for the same energy levels and donor distance.

This is due to the fact that in this state the electron density distribution is initially (in the absence of external fields) spherical symmetric. Correspondingly, the electron can be bound with its image at large values of $\rho$ and situated between the donor and the interface. ${ }^{28}$

With an increase of magnetic field, the left lobe for the $2 p_{z}$ state reconstructs as we see in Fig. 5(a) for $\gamma=0.2$. The probability densities finally exhibit three extrema, with two main lobes on the left and right sides of the donor in the $z$ direction. For the $2 s$ state, a weak magnetic field is enough to shift the maximum probability from the donor to both sides of the donor and creates two symmetric lobes around the donor. One can see in Fig. 5(a) that the electron probability density for both cases, of the $2 s$ and $2 p_{z}$ states, is strongly squeezed in the $\rho$ direction by a strong magnetic field, which shows the validity of the choice of the trial function with the one-dimensional factor responsible for the electron-donor interaction. Notice that for the $2 s$ state the electron is squeezed also in the $z$ direction at $\gamma=2$ (the $2 p_{z}$ state may have similar behavior for extremely large magnetic field).

This tendency is clearly seen in Fig. 6, where the effective radius $\rho$ of the electron from the donor for $d=10 a_{B}$ monotonically decreases with magnetic field for the ground as well as for the excited states, leading also to a decrease of the absolute value of the average distances $\langle z-d\rangle$. The average distances for the excited states change more quickly than for the ground state due to the larger extension of the wave function of these states, allowing for a larger compressibility of the excited states.

As shows the inset of Fig. 6, for $d=10 a_{B}$, in contrast with $2 s, 2 p_{ \pm}$states, the $2 p_{z}$ state has initially (in the absence of magnetic field) a positive average distance from the donor. While all these states become more localized around the donor with increasing magnetic field, i.e., $|\langle z-d\rangle|$ monotonically decreases, we observe a change in the sign of $\langle z-d\rangle$ for the $2 p_{z}$ state. So, the electron shifts towards the interface, i.e., to the left side of the donor. The reason is that the $2 p_{z}$ state has three lobes and it is strongly influenced by the presence of the 

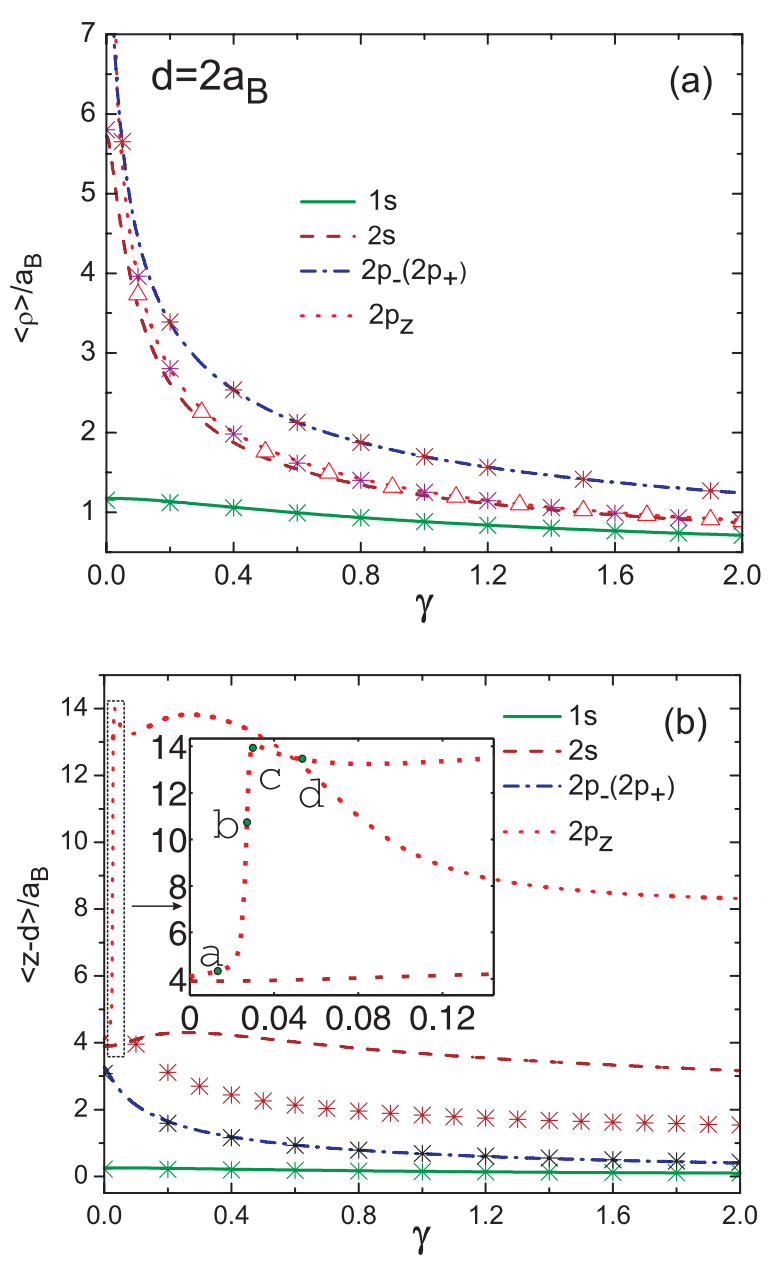

FIG. 7. (Color online) (a) Effective radius $\langle\rho\rangle$ of the electron, in case of small donor distance $d=2 a_{B}$, as a function of the magnetic parameter $\gamma$, for five different energy levels: $1 s$ (solid line), $2 p_{-}$ $\left(2 p_{+}\right)$(dotted-dashed curve), $2 s$ (dashed curve), $2 p_{z}$ (dotted curve). Variational results are indicated by stars for $1 s, 2 s, 2 p_{-}\left(2 p_{+}\right)$states and by triangles for the $2 p_{z}$ state. (b) Average distance of the electron from the donor $\langle z-d\rangle$ for $d=2 a_{B}$. The marked area is enlarged in the inset.

metallic interface. A crossing of the $\langle z-d\rangle$ curves for the $2 s$ and $2 p_{z}$ can be observed at the magnetic parameter $\gamma \simeq 0.885$.

Notice that the $2 s$ state has a larger $\langle\rho\rangle$ value than the $2 p_{z}$ state for small value of $\gamma$. The reason is that the $2 s$ level has a secondary extremum at the left side of the donor, where the image of the electron pulls the electron wave function away from the donor, which results in a smaller Coulomb interaction with the donor (in comparison with to the $2 p_{z}$ state) and a worse localization in the radial direction.

Figures 6 and 7 show that the $2 p_{-}$and $2 p_{+}$states have the same effective radius $\langle\rho\rangle$ and $\langle z-d\rangle$ for all values of $\gamma$ as the angular momentum gives only a different Zeeman energy in the Hamiltonian. We found that the $1 s$ state which has the lowest energy always has the smallest effective radius $\langle\rho\rangle$. Moreover, it has a nearly zero average distance $\langle z-d\rangle$ from the donor, which means it is rather symmetric with respect to the donor in the $z$ direction.

For $d=2 a_{B}$, as a result of the strong repulsion from the interface, the electron distribution for the $2 s$ and $2 p_{z}$ states is strongly shifted to the right side as shown in Fig. 5(b). In the absence of a magnetic field, both the $2 s$ and $2 p_{z}$ states show a secondary extremum close to the interface. With increase of the magnetic field, a new lobe appears in the $2 p_{z}$ state at the right side of the donor. Similarly as in Fig. 5(a), here the $2 s$ and $2 p_{z}$ states will finally have, respectively, two and three lobes. However, the density distribution is more symmetric with respect to the donor along the $z$ direction for the case $d=10 a_{B}$. Notice that for the $2 p_{z}$ state in weak magnetic fields the largest probability density is in the left lobe, and in the third, i.e., the new lobe that appears at large magnetic fields, but is never located in the middle lobe (where the $2 s$ state is mainly localized at large magnetic field), which is also the reason why we have a steep increase of $\langle z-d\rangle$ in Fig. 7(b).

Figure 7(b) shows the results for the average electron donor distance $\langle z-d\rangle$ for the different energy levels when $d=2 a_{B}$. We notice that all levels have a positive value of $\langle z-d\rangle$ as a result of the repulsion from the interface, and the expectation value $\langle z-d\rangle$ is largest for the $2 p_{z}$ state. In the case of a donor interface distance $d=2 a_{B}$, the results of $\langle\rho\rangle$ for the energy levels are shown in Fig. 7(a). In weak magnetic fields, with the parameter $\gamma$ of about 0.033 , the curve $\langle\rho\rangle$ for the $2 p_{z}$ state crosses the $2 p_{ \pm}$one. In weaker fields, $\langle\rho\rangle$ for the $2 p_{z}$ becomes larger than for the $2 p_{ \pm}$state. This can be explained by the fact that at such small distance $d=2 a_{B}$, the $2 p_{z}$ state is almost ionized due to the close presence of the repulsing interface, and correspondingly the electron is spread out near the interface. Notice that values of $\langle\rho\rangle$ obtained variationally coincide with the corresponding exact FEM results. We found that in intermediate and strong magnetic fields, the variational results for $\langle\rho\rangle$ of $2 s$ and $2 p_{z}$ states are very close to the corresponding magnetic length, which additionally shows the validity of the adiabatic approach. We obtained good results for the $\langle z-d\rangle$ expectation value for the variational $1 s$ and the $2 p_{ \pm}$states, while for the $2 s$ one there is no good agreement with the FEM results. For the $2 p_{z}$ state, the error is even larger due to the fact that the binding (i.e., the Coulomb interaction) energy obtained using Eq. (9) is not in good agreement with the corresponding FEM results (Fig. 4).

As we mentioned before, the probability density increases with increasing magnetic field [see Fig. 5(b)] in the region away from the donor (outer lobe), thus we have an increasing electron-donor distance $\langle z-d\rangle$ along the $z$ direction until the electron is mainly localized in the outer lobe. After that, a further increase of the magnetic field squeezes the wave function of the electron in the radial direction, which results in a decrease of the electron-donor distance $\langle z-d\rangle$. However, this tendency for the state $2 p_{z}$ is more complicated. As we can see clearly from the inset of Fig. 7(b), $\langle z-d\rangle$ for the state $2 p_{z}$ increases rapidly at first, and after it reaches its first extremum, $\langle z-d\rangle$ decreases with increasing $\gamma$.

The nonmonotonic peculiar behavior for the average $\langle z-$ $d\rangle$ in the $2 p_{z}$ state when $d=2 a_{B}$ is shown in Fig. 7. This peculiarity can be explained in the following way. Initially (in the absence of the interface and the magnetic field), the $2 p_{z}$ state is more extended in the $z$ direction. In this case, when the donor is located near the interface with $d=2 a_{B}$, due to the presence of the metallic gate, the $2 p_{z}$ state becomes very shallow. In order to increase the binding energy, the 


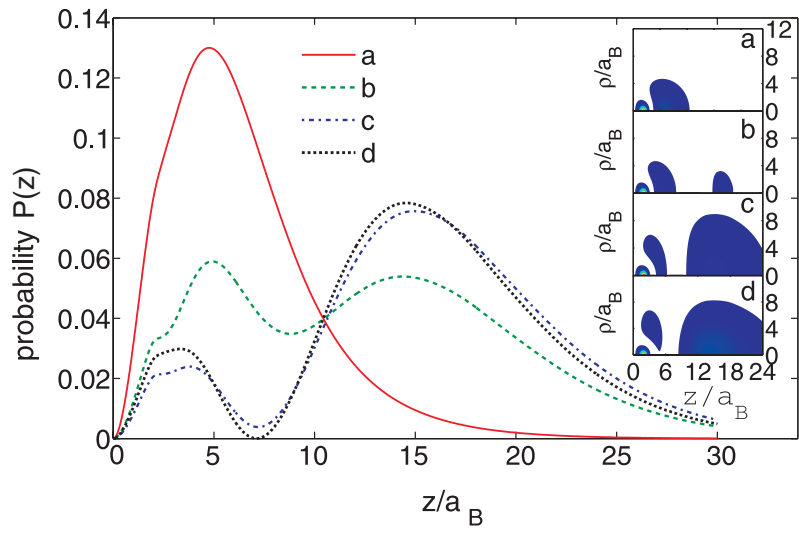

FIG. 8. (Color online) Probability of the electron $\mathrm{P}(z)$ in the plane parallel to the interface when $d=2 a_{B}$ (at the distance $|z-d|$ from the donor) for the energy level $2 p_{z}$. Here a, b, c, and d correspond to the points in the inset of Fig. 7(b). Inset: Probability densities of the electron at these points in the $\rho-z$ plane.

electron in the $2 p_{z}$ state interacts stronger with its image and, correspondingly, its probability density is spread out along the interface [in order to be far away from the donor image, see Fig. 5(b)]. Due to the shallowness of this state, the magnetic field will have a stronger effect, resulting in an easy squeeze of the electron closer to the donor image. In the close vicinity of the interface $\left(d=2 a_{B}\right)$, already the small squeezing of the wave function in the $\rho$ direction can be critical. This squeezing leads to a dominant strong repulsion from the donor image in the $z$ direction and to the abrupt increase of the average distance $\langle z-d\rangle$ for small values of gamma, as we see in Fig. 5(b). Further increase of the magnetic field does not lead to a sufficient decrease in the $\rho$ direction (Fig. 7), but leads to a squeezing in the $z$ direction.

For a better understanding of the electron behavior in the magnetic field, when the donor is near the interface $\left(d=2 a_{B}\right)$, we calculated the probability $\mathrm{P}(z)$ for an electron in the $2 p_{z}$ state to be at a distance $z$ from the interface:

$$
\mathrm{P}(z)=\iint \mathrm{P}_{\mathrm{b}}\left(\rho, z^{\prime}\right) \delta\left(z-z^{\prime}\right) \rho d \rho d z^{\prime},
$$

where $\mathrm{P}_{\mathbf{b}}\left(\rho, z^{\prime}\right)$ is the probability to find the electron at $\left(\rho, z^{\prime}\right)$ and $\delta\left(z-z^{\prime}\right)$ is the Dirac delta function. The results for $\mathrm{P}(z)$ are shown in Fig. 8, where a (red solid curve), b (green dashed curve), c (blue dotted-dashed curve), and d (black dotted curve) correspond to points shown in the inset of Fig. 7(b). The inset of Fig. 8 gives the probability densities of the $2 p_{z}$ state for these four points. The state has two lobes in the absence of magnetic field, and when $\gamma$ changes from a to $b$, a third lobe appears and becomes largest at the point $\mathrm{c}$; this can be seen clearly from the plot of $\mathrm{P}(z)$. It only has one peak (two overlapping peaks but too close to distinguish from each other) which is located in the region with small $z$ at small magnetic field. By increasing the magnetic field, another peak appears in the region with large value of $z$, and it becomes the larger peak and moves towards the region with large value of $z$ at point $\mathrm{c}$ where the magnetic field is larger. However, this peak does not continue to move to the outer region at point $d$, but slightly pushed towards the left (this can also be seen in the lowest figure of the inset of Fig. 8). In that case, the magnetic field just slightly squeezes the electron wave function in the radial direction, which may decrease the electron-donor distance and thus increase the Coulomb interaction between them. As a result, a slightly decreasing tendency of $\mathrm{P}(z)$ becomes possible. However, when the magnetic field reaches a certain value, the outer lobe has the largest probability and $\mathrm{P}(z)$ can decrease again until it reaches its second extremum.

\section{CONCLUSIONS}

In this paper, we studied, within the effective mass approximation, the lowest-energy states of an electron bound to a donor which is located near a semiconductor-metal interface in the presence of a perpendicular magnetic field. We used a variational approach, which takes all image charges that result from the metallic interface into account. A numerical "exact" calculation based on the finite element method was also presented for comparative purposes.

We proposed trial wave functions that decrease exponentially in a small magnetic field and has a Gaussian behavior in sufficiently large magnetic field. The variational calculation leads to a small relative underestimation of the energy when compared to the "exact numerical" FEM results in both small and large magnetic fields. The numerical and variational calculations show a clear different influence of the magnetic field on the electron energy levels for different values of donor-interface distance $d$. We studied the energetics of $1 s$, $2 s, 2 p_{z}, 2 p_{-}$, and $2 p_{+}$states as a function of the magnetic field for different values of $d$. We found that for large value of $d$, the lowest four states are almost degenerate as is the case of a hydrogenic atom in the absence of a magnetic field while for small value of $d$, only the $2 p_{-}$and $2 p_{+}$states are degenerate.

The $1 s, 2 p_{-}$, and $2 p_{+}$states have no significant qualitative changes in the shape of the wave functions when increasing the magnetic field, except that they become more localized around the donor. However, the wave functions of the $2 s$ and $2 p_{z}$ states are strongly modified in the presence of a magnetic field, and these changes are quite different for different values of the donor-interface distance $d$ due to the effect of the image potential and the repulsion from the oxide interface. We show that the average electron-donor distance can be tuned by an external magnetic field, and that it exhibits peculiar and interesting behavior for small and large values of the donor-interface distance.

In the absence of a magnetic field, we found in our previous works (Refs. 26 and 28) that in the case of a semiconductor-metal interface, the dielectric mismatch effect may change the energy of the neutral $D^{0}$ center considerably. The screening effect due to the induced image charges in the metallic gate increases the energy, i.e., makes the $D^{0}$ center shallower. In the presence of a magnetic field, the contribution of the image charges decreases. This occurs due to the fact that the magnetic field squeezes the electron wave function closer to the donor. In increasing magnetic field, the image charges of the donor and the electron approach each other. As a consequence, the prevailed repulsive electron-donor image interaction is suppressed by the attractive electron- electron image interaction and the screening decreases. For $d=2 a_{B}$, in the absence of magnetic field, the ground-state binding 
energy is smaller by about $20 \%$ than the corresponding value, when we ignore image charges. In the magnetic field with $\gamma=2$ (for $d=2 a_{B}$ ), the effect of images becomes $10 \%$. This is similar for the $2 p_{+}$and $2 p_{-}$states. In the absence of magnetic field, the image effect strongly reduces (almost by $60 \%$ ) the binding energy of the shallow ${ }^{28} 2 s$ and $2 p_{z}$ states. In a strong magnetic field $(\gamma=2)$, the relative difference between the binding energies (when the image charges are taken into account and in their absence) reduces to $50 \%$.

Our findings for the electron wave function, in particular its analytical expression, and the magnetic field dependence of its variational parameters will be useful in one-qubit operation for the calculation of the hyperfine interaction strength and its tuning in Si quantum computer scheme based on $P$ donors located close to the interface. ${ }^{19}$ In the two-qubit operation, the knowledge of the electron wave function in a perpendicular magnetic field allows us to find the exchange coupling between donor electrons at the interface and also to control exchange value by varying the magnetic field.

Our previous calculations, ${ }^{28}$ which were performed within the effective mass approximation, lead in the absence of magnetic field to a decrease in the donor binding energy with decreasing donor-interface distance. Now, in this paper, in the presence of a magnetic field, we obtain a similar behavior for the binding energies of the ground and excited states near the interface. These results are opposite to recent experimental results, where using a STM tip, it was found in Ref. 16 that the binding energy of Si donors in GaAs increases when the donor was brought close to the GaAs interface. In Ref. 17, in particular, it was found that a single $\mathrm{Mn}$ atom at the 110 surface produces a highly localized acceptor-level wave function and that the level occurs deep in the gap due to the loss of coordination at the surface. A deep acceptor level at the surface was also found in the experiment of Ref. 31. This discrepancy must be attributed to the discrete atomic character of the system near the interface, which was not included in the present treatment of the problem.

\section{ACKNOWLEDGMENTS}

This work was supported by the Flemish Science Foundation (FWO-Vl).
*Deceased.

${ }^{\dagger}$ Francois.Peeters@ua.ac.be

${ }^{1}$ M. H. Ruderman, Annu. Rev. Astron. Astrophys. 10, 427 (1972).

${ }^{2}$ H. Friedrich, Phys. Rep. 183, 37 (1989).

${ }^{3}$ D. Cabib, E. Fabri, and G. Fiorio, Nuovo Cimento B 10, 185 (1972).

${ }^{4}$ A. R. P. Rau and L. Spruch, Astrophys. J. 207, 671 (1976).

${ }^{5}$ R. K. Bhaduri, Y. Nogami, and C. S. Warke, Astrophys. J. 217, 324 (1977).

${ }^{6}$ L. I. Shiff and H. Snyder, Phys. Rev. 55, 59 (1939).

${ }^{7}$ B. P. Zakharchenya and R. P. Seisyan, Usp. Fiz, Nauk 97, 193 (1969) [Sov. Phys.-Usp. 12, 70 (1969)].

${ }^{8}$ D. S. Bulyanitsa and P. P. Pavinskii, Vestn. Leningrad. Univ. 22, 75 (1958).

${ }^{9}$ Y. Yafet, R. W. Keyes, and E. N. Adams, J. Phys. Chem. Solids 1, 137 (1956).

${ }^{10}$ R. J. Elliott and R. Loudon, J. Phys. Chem. Solids 15, 196 (1960).

${ }^{11}$ A. G. Zhilich and B. S. Monozon, Fiz. Tverd. Tela 8, 3559 (1966) [Sov. Phys.-Solid State 8, 2846 (1967)].

${ }^{12}$ H. Hasegawa and R. E. Howard, J. Phys. Chem. Solids 21, 179 (1961).

${ }^{13}$ M. Asnin and A. A. Rogachev, Phys. Status Solidi 20, 755 (1967).

${ }^{14}$ M. I. D’yakonov, D. L. Mitchell, and A. L. Efros, Fiz. Tverd. Tela 10, 2562 (1968) [Sov. Phys.-Solid State 21, 2021 (1969)].

${ }^{15}$ J. M. Shi, F. M. Peeters, and J. T. Devreese, Phys. Rev. B 50, 15182 (1994).

${ }^{16}$ A. P. Wijnheijmer, J. K. Garleff, K. Teichmann, M. Wenderoth, S. Loth, R. G. Ulbrich, P. A. Maksym, M. Roy, and P. M. Koenraad, Phys. Rev. Lett. 102, 166101 (2009).
${ }^{17}$ T. O. Strandberg, C. M. Canali, and A. H. MacDonald, Phys. Rev. B 80, 024425 (2009).

${ }^{18}$ J.-M. Tang and M. E. Flatté, Phys. Rev. B 72, 161315(R) (2005).

${ }^{19}$ B. E. Kane, Nature (London) 393, 133 (1998).

${ }^{20}$ G. P. Lansbergen, Y. Ono, and A. Fujiwara, Nano Lett. 12, 763 (2012).

${ }^{21}$ Rajib Rahman, G. P. Lansbergen, Seung H. Park, J. Verduijn, Gerhard Klimeck, S. Rogge, and Lloyd C. L. Hollenberg, Phys. Rev. B 80, 165314 (2009).

${ }^{22}$ Y. L. Hao, A. P. Djotyan, A. A. Avetisyan, and F. M. Peeters, J. Phys.: Condens. Matter 23, 115303 (2011).

${ }^{23}$ M. J. Calderón, B. Koiller, and S. Das Sarma, Phys. Rev. B 75, 125311 (2007)

${ }^{24}$ S. R. Schofield, N. J. Curson, M. Y. Simmons, F. J. Ruess, T. Hallam, L. Oberbeck, and R. G. Clark, Phys. Rev. Lett. 91, 136104 (2003).

${ }^{25}$ J. K. Garleff, C. Celebi, W. Van Roy, J.-M. Tang, M. E. Flatté, and P. M. Koenraad, Phys. Rev. B 78, 075313 (2008).

${ }^{26}$ A. F. Slachmuylders, B. Partoens, F. M. Peeters, and W. Magnus, Appl. Phys. Lett. 92, 083104 (2008).

${ }^{27}$ Bin Li, A. F. Slachmuylders, B. Partoens, W. Magnus, and F. M. Peeters, Phys. Rev. B 77, 115335 (2008).

${ }^{28}$ Y. L. Hao, A. P. Djotyan, A. A. Avetisyan, and F. M. Peeters, Phys. Rev. B 80, 035329 (2009); 83, 199904(E) (2011).

${ }^{29}$ E. P. Pokatilov and M. M. Rusanov, Fiz. Tverd. Tela. 10, 3117 (1969) [Sov. Phys.-Solid State 10, 2458 (1969)].

${ }^{30}$ A. Latgé, N. Porras-Montenegro, and Luiz E. Oliveira, Phys. Rev. B 45, 9420 (1992).

${ }^{31}$ Kitchen, A. Richardella, J.-M. Tang, M. E. Flatté, and A. Yazdani, Nature (London) 442, 436 (2006). 